\title{
Comparison of 5 Different Rat Models to Establish a Standard Animal Model for Research Into Interstitial Cystitis
}

\author{
Phil Hyun Song ${ }^{1, *}$, So Young Chunn ${ }^{2, *}$, Jae-Wook Chung ${ }^{3}$, Yeon Yong Kim² ${ }^{2}$, Hyo Jung Lee², Jun Nyung Lee ${ }^{3}$, Yun-Sok Ha ${ }^{3}$, \\ Eun Sang $\mathrm{Yoo}^{3}$, Tae Gyun Kwon ${ }^{3}$, Jeongshik Kim², Dae Hwan Kim5, Bum Soo Kim³ \\ ${ }^{1}$ Department of Urology, Yeungnam University College of Medicine, Daegu, Korea \\ ${ }^{2}$ BioMedical Research Institute, Kyungpook National University Hospital, Daegu, Korea \\ ${ }^{3}$ Department of Urology, Kyungpook National University School of Medicine, Daegu, Korea \\ ${ }^{4}$ Department of Pathology, Central Hospital, Ulsan, Korea \\ ${ }^{5}$ Department of Laboratory Animal Research Support Team, Yeungnam University Hospital, Daegu, Korea
}

Purpose: We evaluated 5 different rat models using different agents in order to establish a standard animal model for interstitial cystitis (IC) in terms of the functional and pathologic characteristics of the bladder.

Methods: Five IC models were generated in 8-week-old female Sprague-Dawley rats via transurethral instillation of 0.1M hydrogen chloride ( $\mathrm{HCl}$ ) or 3\% acetic acid (AA), intraperitoneal injection of cyclophosphamide (CYP) or lipopolysaccharide (LPS), or subcutaneous injection of uroplakin II (UPK2). After generating the IC models, conscious cystometry was performed on days 3,7 , and 14 . All rats were euthanized on day 14 and their bladders were obtained for histological and pro-inflammatory-related gene expression analysis.

Results: In the cystometric analysis, all experimental groups showed significantly decreased intercontraction intervals compared with the control group on day 3, but only the LPS and UPK groups maintained significantly shorter intercontraction intervals than the control group on day 14 . The histological analysis revealed that areas with severe urothelial erosion $(\mathrm{HCl}, \mathrm{AA}$, and UPK) and hyperplasia (CYP and LPS), particularly in the UPK-treated bladders, showed a markedly increased infiltration of toluidine blue-stained mast cells and increased tissue fibrosis. In addition, significantly elevated expression of interleukin1b, interleukin-6, myeloperoxidase, monocyte chemotactic protein 1, and Toll-like receptors 2 and 4 was observed in the UPK group compared to the other groups.

Conclusions: Among the 5 different agents, the injection of UPK generated the most effective IC animal model, showing consequent urothelial barrier loss, inflammatory reaction, tissue fibrosis stimulation, and persistent hyperactive bladder.

Keywords: Cystitis, Interstitial; Models, Animal; Rats; Immunization

- Grant/Fund Support: This work was supported by a National Research Foundation (NRF) of Korea grant funded by the Korea government (MSIP) (NRF-2015R1C1A1A01053509). Additional funding was provided by the Ministry of Education (2015R1D1A3A03020378) and Ministry of Science, ICT \& Future Planning under grants (2014R1A1A3049460), (NRF-2014M3A9D3034164), and (2016R1C1B1011180).

- Research Ethics: All rat protocols were preapproved by the institutional animal ethics committee of Yeungnam University College of Medicine (YUMC-2015-028).

- Conflict of Interest: No potential conflict of interest relevant to this article was reported.

\section{- HIGHLIGHTS}

- Injection of uroplakin II generated the effective interstitial cystitis animal model.

- Uroplakin-induced interstitial cystitis rat model maintains bladder overactivity and shows increased expression of inflammatory factors and fibrosis.

Corresponding author: Bum Soo Kim (i) https://orcid.org/0000-0002-4873-3049 Department of Urology, Kyungpook National University Hospital, 130 Dongdeokro, Jung-gu, Daegu 41944, Korea

E-mail: urokbs@knu.ac.kr / Tel: +82-53-200-5855 / Fax: +82-53-421-9618

Submitted: April 23, 2017 / Accepted after revision: July 1, 2017

${ }^{*}$ Phil Hyun Song and So Young Chun contributed equally to this study as co-first authors. 


\section{INTRODUCTION}

Interstitial cystitis (IC) is a chronic inflammatory bladder disorder characterized by pelvic pain and urinary symptoms, such as frequency, urgency, and nocturia, without bacterial infection or identifiable pathology $[1,2]$. Owing to these various symptoms, patients with IC typically experience sleep dysfunction, chronic stress, anxiety, depression, and sexual dysfunction [3]. Epidemiological studies have revealed that IC primarily affects women, but this disease can also occur in men and adolescents, with a femaleto-male ratio of 5:1 $[4,5]$. The etiology of IC is not completely understood; inflammatory, neurogenic, autoimmune, vascular, and lymphatic disorders have been suggested as potential causes. Further, loss of the glycosaminoglycan layer from superficial urothelial cells and the presence of toxic substances in the urine have been proposed as pathophysiological mechanisms [6]. This poor understanding of the pathophysiology of IC makes it difficult to develop effective treatment modalities. Although oral medicines such as pentosan polysulfate sodium and amitriptyline, hydrodistention, and intravesical instillation are currently used in clinical practice, no standard treatment method yet exists for IC.

Recently, several studies have proposed new potential therapeutic options, such as stem cell therapy, for IC $[7,8]$. In addition, many in vivo studies of IC have been performed. To confirm the efficacy and safety of new treatment modalities, it is necessary to establish a proper animal model for in vivo evaluation. Although more than 20 existing animal models of IC have been evaluated, there is no standard animal model of IC with features similar to those of the disease in humans. Most animal models have been generated by inducing bladder inflammation with or without epithelial damage via the intravesical instillation of chemical toxins or irritants or by the systemic injection of chemical agents, viruses, or antigens [8-16]. Although many animal models generated by various methods have been used to study IC over the last several decades, recent studies have attempted to establish a more appropriate IC animal model, suggesting several potential candidates with characteristics similar to those of the human disease $[8,9,14,16,17]$. Although these animal models show several phenotypes of IC, such as frequent voiding, alteration of bladder cells, and higher expression of pro-inflammatory genes, it has not been confirmed whether these characteristics persist for long periods without natural healing or which models are the most similar to the human disease. Therefore, we analyzed and compared the 5 most commonly used and promising rat models to determine the optimal
IC animal model in terms of the functional and pathologic characteristics of the bladder.

\section{MATERIALS AND METHODS}

\section{Generation of IC Rat Models}

All rat protocols were preapproved by the institutional animal ethics committee of Yeungnam University College of Medicine (YUMC-2015-028). To induce the IC models, thirty 8-week-old female Sprague-Dawley rats were subjected to 5 different agents: hydrogen chloride $(\mathrm{HCl})$, acetic acid (AA), cyclophosphamide (CYP), lipopolysaccharide (LPS), and uroplakin (UPK) II. Each group included 5 rats, and the remaining 5 rats underwent a sham operation to constitute the control (CON) group. All rats were anesthetized by the intramuscular injection of $16 \mathrm{mg} / \mathrm{kg}$ of Rompun and $0.04 \mathrm{mg} / \mathrm{kg}$ of Zoletil, and an abdominal incision was made. The bladder was exposed and a small incision at the bladder dome area was made. Next, one of the ends of a polyethylene-50 tube (Natsume Seisakusho Co., Tokyo, Japan) was placed inside the bladder, and the bladder was closed to be water-tight. The contralateral end of the polyethylene tube was passed through the subcutaneous layer of the left flank area and pulled out at the posterior neck area. After confirming that the tube was not obstructed and that urine drained well, without leakage, through the bladder incision site, the polyethylene-50 tube was fixed and the abdominal wound was closed. To prevent procedure-related infections, an intramuscular injection of $15 \mathrm{mg} / \mathrm{kg}$ of cefprozil was administered, and the animals were observed in warm cages until they woke up completely.

Three days after tube placement, IC rat models were induced. In the $\mathrm{HCl}$ group, $0.1 \mathrm{M} \mathrm{HCl}$ (Sigma-Aldrich, St. Louis, MO, USA) was slowly instilled via the urethra, using a 26-G angiocatheter (Sewoon Medical Co., Cheonan, Korea), followed by neutralization and washing with saline [8]. In the AA group, 50 $\mu \mathrm{L}$ of 3\% AA (Sigma-Aldrich) was transurethrally infused [17]. In the CYP group, $80 \mathrm{mg} / \mathrm{kg}$ of CYP (Sigma-Aldrich) in sterile normal saline $(0.9 \% \mathrm{NaCl})$ was administered intraperitoneally, and the injections were given 4 times every other day [16]. In the LPS group, $10 \mathrm{~mL} / \mathrm{kg}$ of LPS (Sigma-Aldrich) dissolved in sterile normal saline was administered via intraperitoneal injection [14]. In the UPK group, $200 \mu \mathrm{L}$ of an emulsion of equal volumes of phosphate-buffered saline (PBS) and glycerol with $200 \mu \mathrm{g}$ of UPK2 (MyBiosource, San Diego, CA, USA), which is a recombinant protein produced by Escherichia coli via mouse base sequencing, was injected subcutaneously into the abdomi- 
nal flank area of the rats [9]. For the CON group, PBS was instilled transurethrally using an angiocatheter. The animals were briefly observed daily for signs of pain (abnormal behavior or a hunched appearance).

\section{Cystometry}

After generating the IC models in each group, conscious cystometry was performed on days 3,7 , and 14 . Rats were placed under restriction in a specially designed cage without anesthesia, and the exposed polyethylene-50 tube was connected to a pressure transducer. Sterile warm saline was infused at a rate of $40 \mu \mathrm{L} / \mathrm{min}$ via a syringe pump for 30 minutes. Intravesical pressure and intercontraction intervals (or voiding intervals) were measured and analyzed using the Power Lab system (AD Instruments Pty., Ltd., Bella Vista, NSW, Australia).

\section{Histological Analysis}

For the histological analysis, half of each bladder was fixed in $4 \%$ paraformaldehyde 14 days after generating the animal models (the 5 rats in each group were euthanized). The paraffin-embedded samples were cut into 5 - $\mu \mathrm{m}$ sections for hematoxylin and eosin (H\&E), toluidine blue, and Masson trichrome staining for histology and analyses of mast-cell infiltration and fibrosis, respectively. For toluidine blue staining, bladder sections were washed with xylene to remove the paraffin, and then the slides were treated with an ethanol series. The slides were stained with toluidine blue for 4 minutes, treated with an ethanol series, and then mounted. Mast cells were measured per square millimeter, and the lamina propria was used as the observational histological position. For the Masson trichrome staining, deparaffinized and rehydrated sections were refixed in Bouin solution, stained in Weigert iron hematoxylin working solution and Biebrich scarlet-acid fuchsin solution, and differentiated in a phosphomolybdic-phosphotungstic acid solution until the collagen was red. The sections were transferred to aniline blue solution and differentiated in 1\% AA solution. The collagen fibers were stained as a blue color.

\section{Gene Expression Analysis}

The remaining half of each bladder was prepared for real-time polymerase chain reaction (PCR) analysis. Total RNA was isolated using the RNeasy Mini Kit (QIAGEN, Valencia, CA, USA) and complementary DNA was prepared using ReverseTranscription Reagents (Applied Biosystems, Carlsbad, CA, USA) according to the manufacturer's instructions. The follow- ing primers were used: glyceraldehyde 3-phosphate dehydrogenase $(G A P D H)$ : sense TGTGTCCGTCGTGGATCTGA and antisense CCTGCTTCACCACCTTCTTGA; interferon gamma (IFNG): sense CTCGAACTTGGCGATGCTCA and antisense TCGAATCGCACCTGATCACT; interleukin-1b (IL1B): sense CGTCATCATCCCACGAGTCA and antisense GATGAGGACCCAAGCACCTT; IL6: sense AGTCTCCTCTCCGGACTTGT and antisense AGAGACTTCCAGCCAGTTGC; myeloperoxidase (MPO): sense CGCAATTTGGTTCTGGCGAT and antisense ACCTACCCCAGTACCGATCC; monocyte chemotactic protein $1(M C P-1)$ : sense CAGCCGACTCATTGGGATCA and antisense TAGCATCCACGTGCTGTCTC; Toll-like receptor 2 (TLR2): sense TGACGGCCTGTATCCCTGTA and antisense CCCTGCTCTTTCTCACAGCA; TLR4: sense CCAGAGCGGCTACTCAGAAA and antisense TCCACAAGAGCCGGAAAGTT; and IL17A: sense AGAGTCCAGGGTGAAGTGGA and antisense CAAACGCCGAGGCCAATAAC. PCR was carried out in a real-time PCR machine and analyzed using 7300 System SDS Software (Applied Biosystems). The conditions for PCR using the SYBR Green PCR Master Mix (Bio-Rad, Hercules, CA, USA) were $95^{\circ} \mathrm{C}$ for 10 minutes, followed by 45 cycles of $95^{\circ} \mathrm{C}$ for 10 seconds, $58^{\circ} \mathrm{C}$ for 50 seconds, and $72^{\circ} \mathrm{C}$ for 20 seconds. To analyze the relative changes in gene expression, the $C_{t}$ value for the target gene was normalized to its endogenous control and transformed to a relative gene expression value using the $2^{-\Delta \Delta \mathrm{Ct}}$ method.

\section{Statistical Analysis}

The data were presented as the mean \pm standard deviation. Differences in the cystometry, mast cell count, and real-time quantitative PCR results were analyzed by 1-way analysis of variance (ANOVA). A P-value of $<0.05$ was considered to indicate statistical significance. If the value was found to be significant by ANOVA, the Tukey post hoc test was conducted.

\section{RESULTS}

During the whole period after generating the IC model, none of the animals showed any signs of severe pain, such as abnormal behavior or a hunched appearance. To compare bladder function, such as voiding frequency and nonvoiding bladder contractions, we performed conscious cystometric analysis in all animal models, including the CON group. In the cystometric analysis, the intervals between normal voiding events were measured. On day 3 , the mean intercontraction intervals of all 
experimental groups were significantly shorter than that of the CON group; $72.3 \pm 17.5,57.3 \pm 10.1,65.0 \pm 13.5,87.7 \pm 9.8$, $50.0 \pm 5.2$, and $161.3 \pm 8.8$ seconds in the HCl, AA, CYP, LPS, UPK, and $\mathrm{CON}$ groups, respectively $(\mathrm{P}=0.003)$. However, on day 7 , the mean intercontraction intervals of all groups were prolonged; in particular, those of the AA (117.7 \pm 4.3 seconds) and CYP (146.3 \pm 15.1 seconds) groups were significantly longer than those of the $\mathrm{HCl}(83.3 \pm 18.3$ seconds $)$ and UPK $(84.3 \pm 11.0$ seconds $)$ groups $(\mathrm{P}=0.012)$. Additionally, on day 14 , the UPK $(67.6 \pm 6.9$ seconds) and LPS (80.3 \pm 10.1 seconds) groups showed significantly shorter voiding intervals than the CON group (141.7 $\pm 16.7 \mathrm{sec}-$ onds) or the other experimental groups (AA, 117.3 \pm 9.7 seconds; CYP, $153.3 \pm 17.2$ seconds; and $\mathrm{HCl}, 126.3 \pm 21.6$ seconds) $(\mathrm{P}=0.009)$. Irregular nonvoiding bladder contractions were only observed in the UPK and $\mathrm{HCl}$ groups on days 3, 7, and 14 (Fig. 1).

In the $\mathrm{H} \& \mathrm{E}$ histological examination (Fig. 2A), the CON bladders were intact and consisted of 3-5 layers (estimated by nucleus staining with hematoxylin): the regular transitional epithelium, the normal basement membrane, the lamina propria, and the smooth muscle. Although the infiltration of inflammatory cells was rarely observed, the experimental groups showed significant intraepithelial inflammation with severe damage to the bladder tissues. The $\mathrm{HCl}, \mathrm{AA}$, and UPK groups showed urothelial thinning and cellular loss or erosion, while the CYP and LPS groups showed increased numbers of urothelial cells and abnormally thick re-epithelialization (urothelial hyperplasia) after denudation. The number of cellular layers of the reepithelialized groups increased from 5 to 10.

Mast cell infiltration was observed by toluidine blue staining
(Fig. 2B). In the CON bladders, mast cells were rarely located in the lamina propria, the mean number of observed mast cells was $31 \pm 6 / \mathrm{mm}^{2}$, and the cells contained purple granules. The experimental groups showed a greater inflammatory response (mild to moderately dense mixed lymphocytic infiltration with mast cells and eosinophils) compared to the $\mathrm{CON}$ group because of the loss of urothelial barrier function. The numbers of mast cells in the lamina propria in the HCl, AA, CYP, LPS, and UPK groups was $96.5 \pm 10.5,39.5 \pm 1.5,160.5 \pm 23.5,102.0 \pm 11$, and $297.5 \pm 13.5 /$ $\mathrm{mm}^{2}$, respectively (Fig. 3). The UPK, CYP, LPS, and $\mathrm{HCl}$ groups showed significantly greater mast cell infiltration than the CON group $(\mathrm{P}<0.001)$, with the largest number of mast cells observed in the UPK group.

Loss of the urothelial barrier and the inflammatory reaction induced tissue fibrosis in the basement membrane, lamina propria, and smooth muscle layer. Masson trichrome staining revealed a modest increase in bladder tissue fibrosis in many of the experimental groups in comparison to the $\mathrm{CON}$ group; the UPK group showed broader and more severe fibrotic density, as indicated by blue coloring, than the other experimental groups (Fig. 2C)

To evaluate the inflammatory reactions, the expression of pro-inflammatory genes was analyzed (Fig. 4). The expression of the IFNG, IL1B, IL6, MPO, MCP1, TLR2, TLR4, and IL17A genes was significantly higher in the experimental groups than in the CON group. The UPK group showed the highest expression of IL1B, IL-6, MPO, MCP1, and TLR2, whereas TLR4 and IL17A were the most highly expressed in the AA group and IFNG was the most highly expressed in the LPS group.

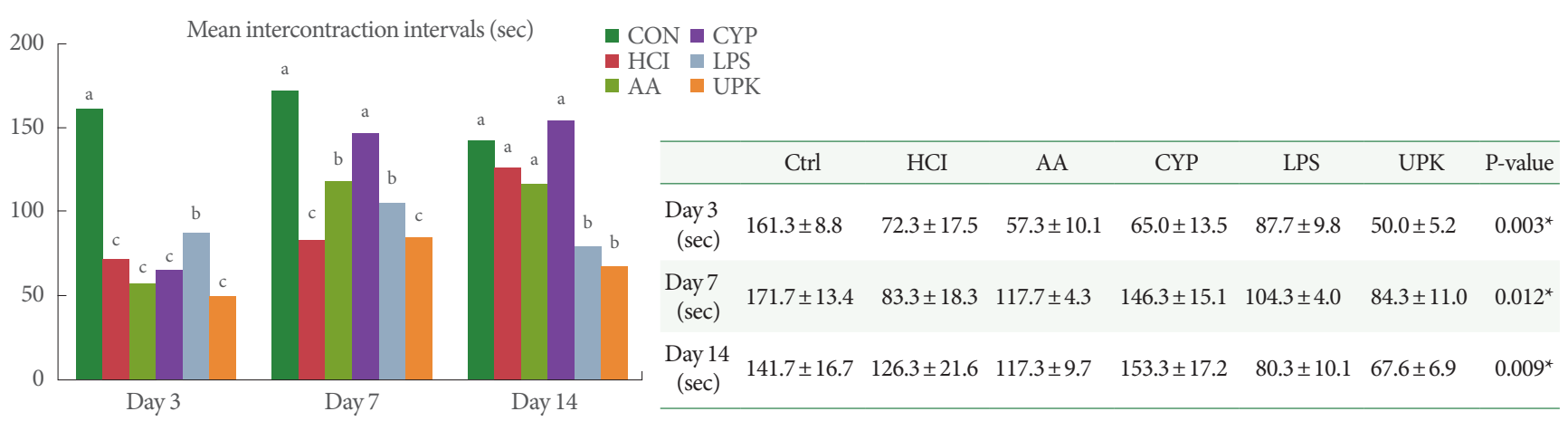

Fig. 1. Comparison of the mean intercontraction intervals measured by conscious cystometric analysis. The mean intercontraction intervals of all experimental groups were significantly shorter than that of the CON group at day 3 , and the UPK and LPS groups maintained shorter voiding intervals until day 14 . Values are presented as mean \pm standard deviation. CON, control; $\mathrm{HCl}$, hydrogen chloride; AA, acetic acid; CYP, cyclophosphamide; LPS, lipopolysaccharide; UPK, uroplakin. The different letters on top of the bars (a, $\mathrm{b}$, and c) mean significant differences between the each group at $\mathrm{P}<0.05$. ${ }^{*}$ Statistical analysis was performed for all groups by analysis of variance, Tukey post hoc test. 


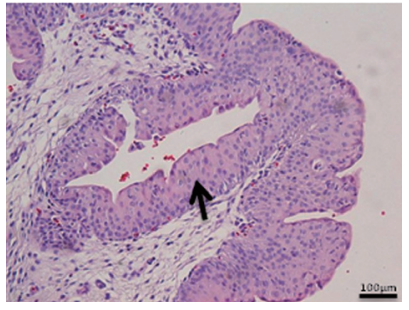

$\mathrm{CON}$

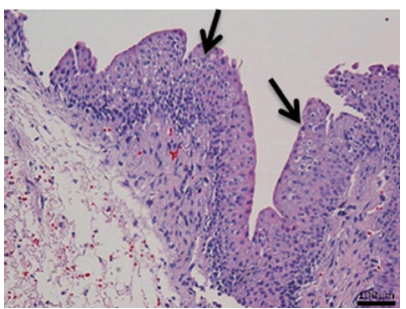

CYP

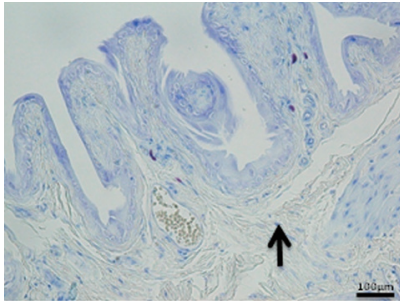

$\mathrm{CON}$

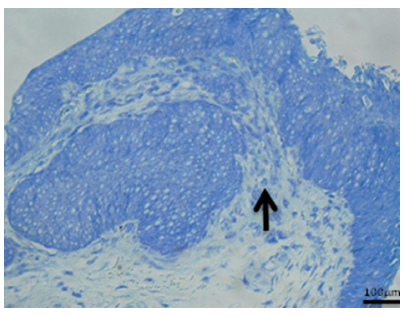

CYP

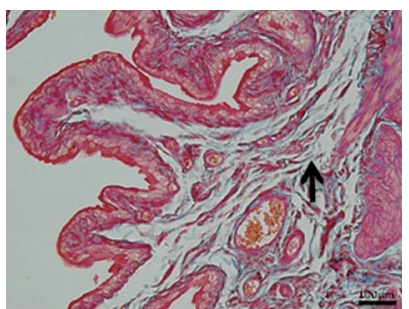

$\mathrm{CON}$

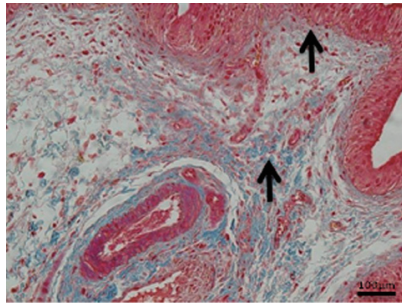

CYP

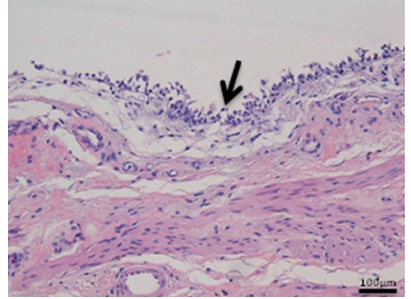

$\mathrm{HCI}$

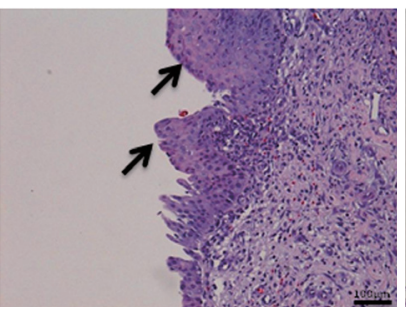

LPS

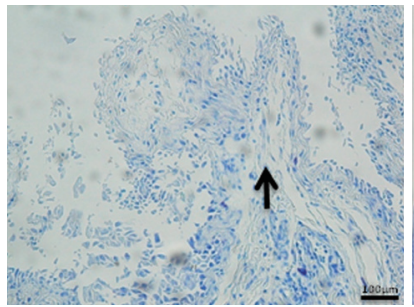

HCI

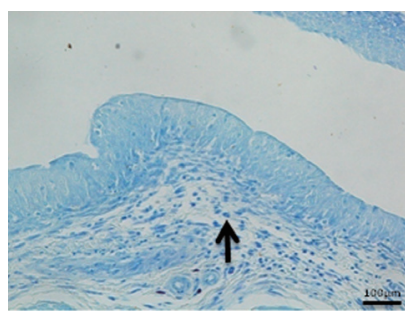

LPS

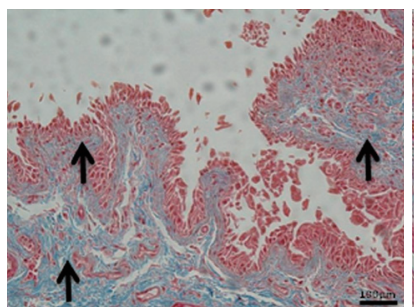

$\mathrm{HCI}$

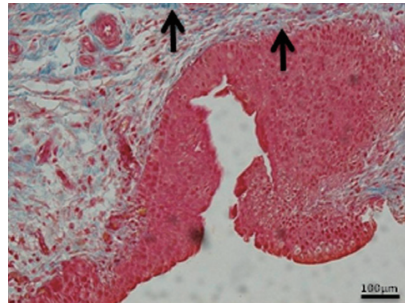

LPS

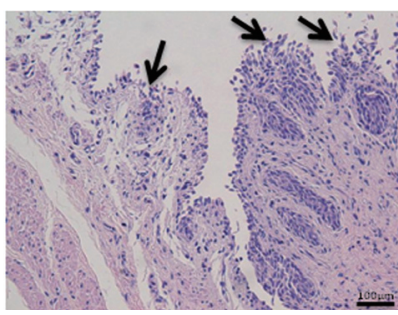

AA
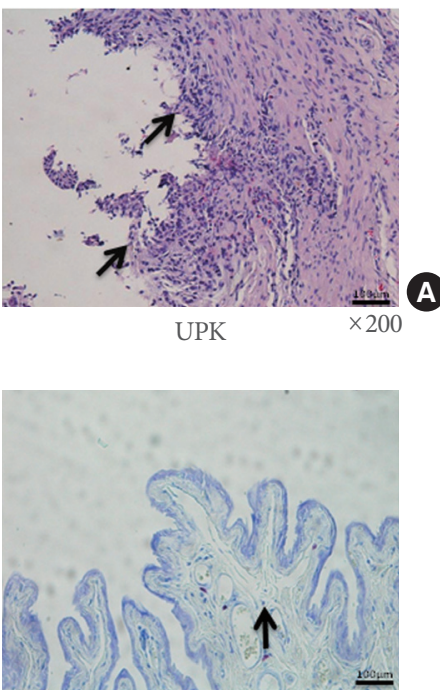

AA

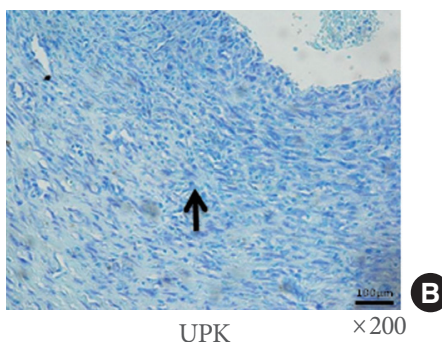

UPK

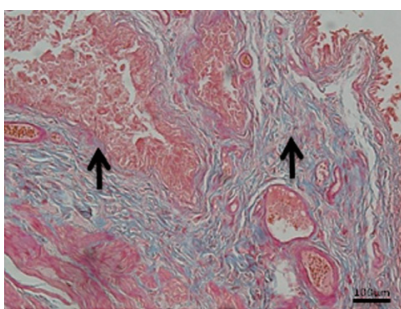

AA

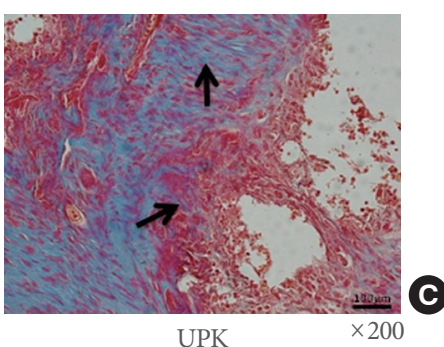

Fig. 2. Histological analysis of bladder tissue. (A) In H\&E staining, the $\mathrm{HCl}, \mathrm{AA}$, and $\mathrm{UPK}$ groups showed urothelial erosions and the CYP and LPS groups showed urothelial hyperplasia (arrow). (B) The UPK group showed the most severe mast cell infiltration (arrow) in the submucosal connective tissue and muscle layer. (C) The UPK group showed the most severe accumulation of fibrous tissue in the submucosa layer (arrow) by Masson trichrome staining. Scale bar, $100 \mu \mathrm{m}$. CON, control; HCl, hydrogen chloride; AA, acetic acid; CYP, cyclophosphamide; LPS, lipopolysaccharide; UPK, uroplakin. 


\section{DISCUSSION}

The pathophysiology of IC is complex and not completely understood. This makes it difficult to develop definitive therapeutic modalities and to establish a standard animal model for translational research. Current approaches have mostly emphasized merging clinical practice and translational research; therefore, it is important to establish proper translational models similar to the disease in humans. Approximately 20 animal models with some similar characteristics to the IC phenotype have been generated over the past several decades [15,18]. From 1980 to 2000,

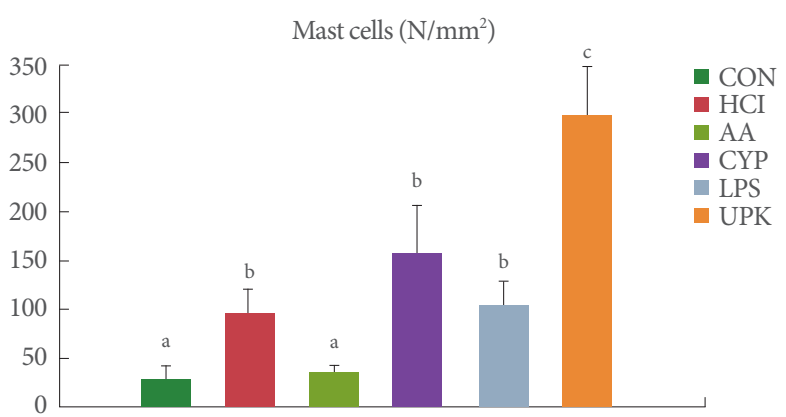

Fig. 3. Comparison of the number of mast cells. The numbers of mast cells were quantified in the bladder sections $\left(\mathrm{mm}^{2}\right)$, and the highest number of mast cells was observed in the UPK group. $\mathrm{CON}$, control; $\mathrm{HCl}$, hydrogen chloride; $\mathrm{AA}$, acetic acid; CYP, cyclophosphamide; LPS, lipopolysaccharide; UPK, uroplakin. The different letters ( $a, b$, and $c$ ) on top of the bars mean significant differences between the each group at $\mathrm{P}<0.05$.

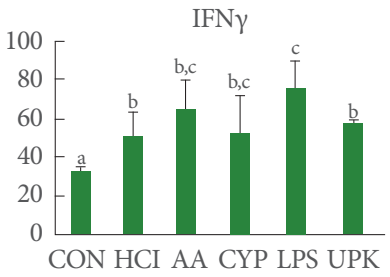

MCP1

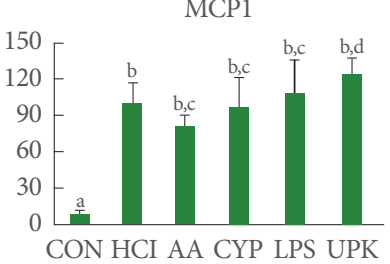

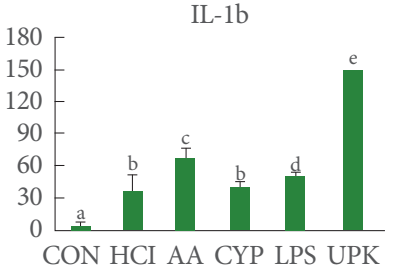

TLR2

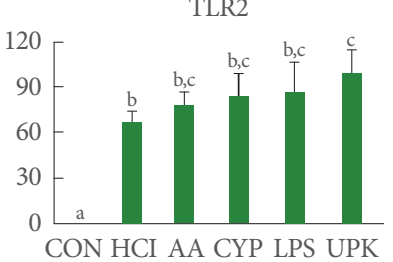

the models were typically prepared by inducing inflammatory conditions in the bladder by intravesical instillation of chemical irritants, such as acetone, acrolein, acid, turpentine, mustard oil, or croton oil [19-22]. In the 2000s, IC animal models were commonly generated by injection of bacterial LPS, systemic administration of CYP, pseudorabies virus infection, or systemic induction of autoimmunity $[9,14,16,23,24]$. Although these materials contributed to the establishment of IC animal models, each agent has several limitations. Most intravesical agents can nonselectively damage the bladder mucosa and glycosaminoglycan layer through various mechanisms that may not be relevant for human IC [18]. Injection of pseudorabies virus may cause acontractile bladder within 5 days of virus injection, and therefore this model does not accurately reflect human IC [24].

To generate a suitable animal model, it is necessary to understand the pathophysiology of the target disease. Although the exact pathogenesis of IC remains unclear, several mechanisms, such as an increased number of mast cells in the bladder, loss of the glycosaminoglycan layer in the bladder mucosa, and autoimmunity, have been suggested as candidates. Based on these theories, several IC animal models with phenotypes similar to that of the human disease have been generated. Some have been recently generated by intravesical instillation of $\mathrm{HCl}$ or AA, injection of CYP or LPS, or immunization with UPK, considering the convenience of generating and maintaining these animal models. The animal model generated by intravesical instillation using $\mathrm{HCl}$ showed most of the pathophysiological
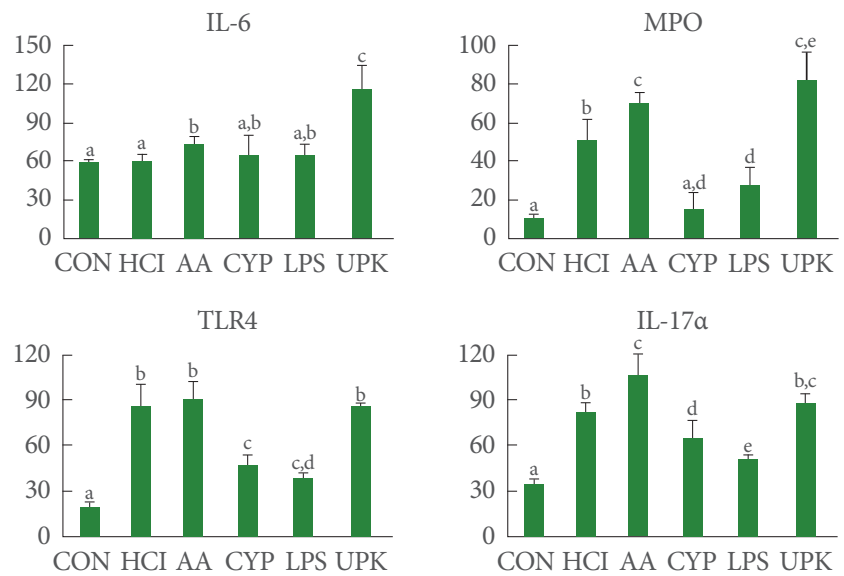

Fig. 4. Inflammatory gene expression analysis by real-time polymerase chain reaction. IL1B, IL6, MPO, MCP1, and TLR2 showed the greatest expression in the UPK group, while the AA group showed the highest expression of TLR4 and IL17A and the LPS group showed the highest expression of IFNG. CON, control; $\mathrm{HCl}$, hydrogen chloride; AA, acetic acid; CYP, cyclophosphamide; LPS, lipopolysaccharide; UPK, uroplakin; IL, interleukin; MPO, myeloperoxidase; MCP1, monocyte chemotactic protein 1; TLR, Toll-like receptor; IFN, interferon. The different letters on top of the bars $(\mathrm{a}, \mathrm{b}$, and $\mathrm{c})$ mean significant differences between the each group at $\mathrm{P}<0.05$. 
manifestations observed in human IC patients, such as epithelial denudation, an abnormal increase in inflammation, neural cell activation, and angiogenesis [8]. The AA-induced IC rodent model showed changes in the expression of two proteins, UPK3 and zonula occludens type I, which are similar to changes that are found consistently in patients with IC [17]. Administration of CYP can induce an increase in pro-inflammatory cytokine expression and in the number of mast cells in the bladder tissue of rodent animal models without signs of massive inflammatory infiltration, tissue hemorrhage, or urothelial damage [16]. Systemic injection or intravesical instillation of LPS was also reported to induce an inflammatory response mediated by the activation of mast cells, the production of cytokines, and the recruitment of leukocytes to the bladder mucosal surface, similar to what is observed in human IC patients [14]. Mice immunized with UPK displayed significantly increased urinary frequency, extensive perivascular infiltration of inflammatory response cells, and elevated gene expression levels of inflammatory cytokines in bladder tissue $[9,24]$.

In our study, all experimental groups showed a higher expression of several inflammatory factors compared to the CON group in the PCR analysis, and increased infiltration of inflammatory cells such as lymphocytes and mast cells in the histological analysis. In addition, bladder hyperactivity was also observed in the early period (day 3 ) in the cystometric analysis of all groups. However, bladder overactivity did not persist for 1 or 2 weeks in the AA, CYP, and $\mathrm{HCl}$ groups, which means that bladder function can be spontaneously restored in animal models generated using these agents. $\mathrm{H} \& \mathrm{E}$ and toluidine blue staining also showed different results in terms of the number of infiltrated inflammatory cells and the thickness of the submucosa layer among the experimental groups. The greatest infiltration of mast cells and the thickest submucosa layer were observed in the UPK group. The most extensive fibrotic changes were observed in the UPK group in Masson trichrome staining. Moreover, in the PCR analysis, the UPK group showed the highest expression of most inflammatory factors, such as $I L 1 B$, IL6, MPO, MCP1, and TLR2. For the IC animal model, it is important to maintain functional bladder overactivity and inflammatory conditions in the bladder for a long period of time to confirm the long-term therapeutic effect of new treatment modalities, and to clarify whether bladder overactivity is resolved by treatment or spontaneously. Taken together, among the IC rat models prepared using 5 different agents, the IC model generated by UPK can be regarded as the most feasible model for
IC-related translational research, and autoimmunity should be further investigated as a promising mechanism of this disease.

An increasing number of reports have described the relationship between IC and autoimmune diseases, such as rheumatoid arthritis, lupus erythematosis, Sjogren syndrome, and ulcerative colitis, as well as increased autoantibodies in the serum of IC patients [25-28]. The UPKs (UPK1, UPK2, and UPK3) comprise a family of integral membrane proteins of the urothelium and are highly expressed in bladder tissue [9]. It has been demonstrated that immunization with UPK2 induced a bladderspecific autoimmune response and sufficient bladder inflammation in mice [9]. These mice displayed significant evidence of frequent voiding and decreased urine output per voiding episode. However, the animal model generated by UPK2 did not show chronic pain, one of the main symptoms of IC. Additionally, a more recent study demonstrated that immunization with UPK3A induced all predominant IC phenotypic characteristics, including increased pelvic pain responses to stimulation with von Frey filaments [24]. Considering these results, UPK3A can be used to generate an IC animal model. We used UPK2 to generate an autoimmune IC model in this study in order to maintain the generated animal models for at least 2 weeks to compare long-term cystometric outcomes. Persistent chronic pain can cause unwanted stressful conditions in animal models, and it is also not appropriate to expose the animals to chronic pain for a long time with respect to the ethics of animal studies.

Although the results of this study suggest that an IC rat model generated by immunization using UPK is feasible for ICrelated research, such a model may not exhibit all the phenotypes of IC because there are several proposals regarding the etiopathogenesis of IC, especially in light of the various subtypes of IC, such as ulcerative and nonulcerative IC. However, this animal model is suitable for IC-related research into the common phenotypes of IC, such as bladder overactivity, inflammatory change of bladder mucosa, higher expression of inflammatory genes in bladder tissue, and the relatively long persistence of these phenotypes. Moreover, our results support autoimmunity as one of the main aspects of the pathogenesis of IC, and further studies of the relationship between autoimmunity and IC will provide insight into the pathophysiology of IC and facilitate the development of new therapeutic modalities.

In conclusion, this study demonstrated that injection of UPK generated the most effective IC animal model among the 5 most commonly used agents. This model maintained bladder overactivity and showed increased expression of inflammatory 
factors and fibrosis. Future studies of the relationship between autoimmunity and the pathophysiology of IC may increase our understanding of the pathogenesis of IC and facilitate the development of definitive therapeutic strategies.

\section{REFERENCES}

1. Bogart LM, Berry SH, Clemens JQ. Symptoms of interstitial cystitis, painful bladder syndrome and similar diseases in women: a systematic review. J Urol 2007;177:450-6.

2. Chancellor MB, Yoshimura N. Treatment of interstitial cystitis. Urology 2004;63(3 Suppl 1):85-92.

3. Kim A, Shin DM, Choo MS. Stem Cell Therapy for interstitial cystitis/bladder pain syndrome. Curr Urol Rep 2016;17:1.

4. Clemens JQ, Meenan RT, O’Keeffe Rosetti MC, Brown SO, Gao SY, Calhoun EA. Prevalence of interstitial cystitis symptoms in a managed care population. J Urol 2005;174:576-80.

5. Sea J, Teichman JM. Paediatric painful bladder syndrome/interstitial cystitis: diagnosis and treatment. Drugs 2009;69:279-96.

6. Sand PK. Proposed pathogenesis of painful bladder syndrome/interstitial cystitis. J Reprod Med 2006;51(3 Suppl):234-40.

7. Adamowicz J, Pokrywczyńska M, Drewa T. Conditioned medium derived from mesenchymal stem cells culture as a intravesical therapy for cystitis interstitials. Med Hypotheses 2014;82:670-3.

8. Song M, Lim J, Yu HY, Park J, Chun JY, Jeong J, et al. mesenchymal stem cell therapy alleviates interstitial cystitis by activating Wnt signaling pathway. Stem Cells Dev 2015;24:1648-57.

9. Altuntas CZ, Daneshgari F, Sakalar C, Goksoy E, Gulen MF, Kavran M, et al. Autoimmunity to uroplakin II causes cystitis in mice: a novel model of interstitial cystitis. Eur Urol 2012;61:193-200.

10. Birder LA, Wolf-Johnston A, Buffington CA, Roppolo JR, de Groat WC, Kanai AJ. Altered inducible nitric oxide synthase expression and nitric oxide production in the bladder of cats with feline interstitial cystitis. J Urol 2005;173:625-9.

11. Bon K, Lichtensteiger CA, Wilson SG, Mogil J. Characterization of cyclophosphamide cystitis, a model of visceral and referred pain, in the mouse: species and strain differences. J Urol 2003;170:1008-12.

12. Fraser MO, Chuang YC, Lavelle JP, Yoshimura N, de Groat WC, Chancellor MB. A reliable, nondestructive animal model for interstitial cystitis: intravesical low-dose protamine sulfate combined with physiological concentrations of potassium chloride. Urology 2001;57(6 Suppl 1):112.

13. Kirimoto T, Nakano K, Irimura K, Hayashi Y, Matsuura N, Kiniwa $\mathrm{M}$, et al. Beneficial effects of suplatast tosilate (IPD-1151T) in a rat cystitis model induced by intravesical hydrochloric acid. BJU Int
2007;100:935-9.

14. Tambaro S, Casu MA, Mastinu A, Lazzari P. Evaluation of selective cannabinoid $\mathrm{CB}(1)$ and $\mathrm{CB}(2)$ receptor agonists in a mouse model of lipopolysaccharide-induced interstitial cystitis. Eur J Pharmacol 2014;729:67-74.

15. Westropp JL, Buffington CA. In vivo models of interstitial cystitis. J Urol 2002;167(2 Pt 1):694-702.

16. Golubeva AV, Zhdanov AV, Mallel G, Dinan TG, Cryan JF. The mouse cyclophosphamide model of bladder pain syndrome: tissue characterization, immune profiling, and relationship to metabotropic glutamate receptors. Physiol Rep 2014;2:e00260.

17. Keay S, Leitzell S, Ochrzcin A, Clements G, Zhan M, Johnson D. A mouse model for interstitial cystitis/painful bladder syndrome based on APF inhibition of bladder epithelial repair: a pilot study. BMC Urol 2012;12:17.

18. Bjorling DE, Wang ZY, Bushman W. Models of inflammation of the lower urinary tract. Neurourol Urodyn 2011;30:673-82.

19. Elgebaly SA, Allam ME, Walzak MP Jr, Oselinsky D, Gillies C, Yamase H. Urinary neutrophil chemotactic factors in interstitial cystitis patients and a rabbit model of bladder inflammation. J Urol 1992;147:1382-7.

20. Kato K, Kitada S, Longhurst PA, Wein AJ, Levin RM. Time-course of alterations of bladder function following acetone-induced cystitis. J Urol 1990;144:1272-6.

21. McMahon SB, Abel C. A model for the study of visceral pain states: chronic inflammation of the chronic decerebrate rat urinary bladder by irritant chemicals. Pain 1987;28:109-27.

22. Sakata T, Smith RA, Garland EM, Cohen SM. Rat urinary bladder epithelial lesions induced by acrolein. J Environ Pathol Toxicol Oncol 1989;9:159-69.

23. Jasmin L, Janni G, Ohara PT, Rabkin SD. CNS induced neurogenic cystitis is associated with bladder mast cell degranulation in the rat. J Urol 2000;164(3 Pt 1):852-5.

24. Izgi K, Altuntas CZ, Bicer F, Ozer A, Sakalar C, Li X, et al. Uroplakin peptide-specific autoimmunity initiates interstitial cystitis/ painful bladder syndrome in mice. PLoS One 2013;8:e72067.

25. Keay S, Zhang CO, Trifillis AL, Hebel JR, Jacobs SC, Warren JW. Urine autoantibodies in interstitial cystitis. J Urol 1997;157:1083-7.

26. Ochs RL. Autoantibodies and interstitial cystitis. Clin Lab Med 1997;17:571-9.

27. van de Merwe JP. Interstitial cystitis and systemic autoimmune diseases. Nat Clin Pract Urol 2007;4:484-91.

28. Lorenzo Gómez MF, Gómez Castro S. Physiopathologic relationship between interstitial cystitis and rheumatic, autoimmune, and chronic inflammatory diseases. Arch Esp Urol 2004;57:25-34. 